\title{
A REFINEMENT OF THE INEQUALITY BETWEEN ARITHMETIC AND GEOMETRIC MEANS
}

\author{
J. M. ALDAZ
}

Abstract. In this note we present a refinement of the AM-GM inequality, and then we estimate in a special case the typical size of the improvement.

Mathematics subject classification (2000): 26D15.

Keywords and phrases: Arithmetic geometric inequality, concentration of measure.

\section{REFERENCES}

[Al] Aldaz, J. M.; A stability version of Hölder's inequality. J. Math. Anal. Appl. 343 (2008), no. 2, 842-852. doi:10.1016/j.jmaa.2008.01.104. Also available at the Mathematics ArXiv: arXiv:math.CA/0710.2307.

[A12] AldAZ, J. M.; Concentration of the ratio between the geometric and arithmetic means. Preprint available at the Mathematics ArXiv: arXiv:0807.4832.

[GluMi] Gluskin, E.; Milman, V.; Note on the geometric-arithmetic mean inequality. Geometric aspects of functional analysis, 130-135, Lecture Notes in Math., 1807, Springer, Berlin, 2003.

[MiSch] Milman, Vitali D.; Schechtman, Gideon; Asymptotic theory of finite-dimensional normed spaces. With an appendix by M. Gromov. Lecture Notes in Mathematics, 1200. Springer-Verlag, Berlin, 1986.

[Web] Webster, R. J.; Convexity (Oxford University Press, 1997). 